\title{
Multivariate Statistical Analysis of Series of Diffraction Patterns
}

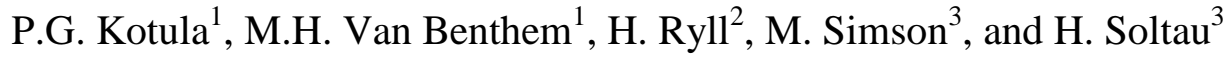 \\ ${ }^{1}$ Sandia National Laboratories, PO Box 5800, MS 0886, Albuquerque, NM 87185-0886, USA \\ ${ }^{2}$ PNSensor GmbH, Otto-Hahn-Ring 6, 81739 Munich, Germany \\ ${ }^{3}$ PNDetector GmbH, Otto-Hahn-Ring 6, 81739 Munich, Germany
}

Commercial-based x-ray hyperspectral imaging (full X-ray spectrum at each pixel in an array versus simple mapping of pre-chosen elements) systems have been available since the mid-1990's [1]. Similar to the evolution of X-ray mapping to hyperspectral imaging, position-resolved electron diffraction is evolving from just orientation information from pre-chosen phases [2] to acquisition of full diffraction patterns at each point in an array. In the case of hyperspectral data, advanced statistical analysis methods have proven efficient and effective at reducing the dimensionality of the data to just a handful of chemically relevant components [3]. An analogous method has been applied to electron backscattered diffraction (EBSD) data [4]. This yielded a dimensional reduction and discrimination of similar space groups (Fd3m versus Fm3m). This was achieved by looking at all the detail in the patterns (not just angles between planes) as well as being robust to mixed patterns from two grains illuminated in one pattern. In the TEM, much progress has been made in orientation imaging by collecting series of diffraction patterns, analyzing data via template matching [5] and also using precession to minimize dynamical effects [6]. In this paper we describe the application of multivariate statistical analysis (MSA) methods to a parallel-beam nano-diffraction image-resolved data set, modifying and building upon the methods developed in [4]. In addition we will also discuss the use of similar methods to analyze data from a direct electron detector in scanning TEM (STEM) mode.

Position-resolved diffraction data were acquired on a FEI Company Titan 80-200 operated at 200kV and equipped with three-condenser lenses and a probe spherical-aberration corrector. Diffraction patterns were acquired on both a Gatan Orius 200D lens-coupled CCD and PNDetector direct electron camera [7]. Figure 1 shows the results of a 30 by 30 real-space pixel $10 \mathrm{~nm}$ parallel nano-beam diffraction data set where each pattern consisted of 512 by 512 reciprocal-space pixels which were subsequently binned 4 by 4 to 128 by 128 pixels for a data set of $30 \mathrm{Mb}$. The data were analyzed with Sandia's Automated eXpert Spectral Image Analysis (AXSIA) MSA software [3] such that the unfolded reciprocal-space pixels were the pixels and the real-space pixels were the channels. The specific analysis performed had the result of making the real-space (pixel) domain simple [8]. From the original 900 diffraction patterns, AXSIA reduced that to 9 of which two in particular are shown in Figure 1. Fig. 1a is the STEM overview image of the sample showing a prominent central grain. Figure $1 \mathrm{~b}$ is the first 64 sorted eigenvalues. Figs. 1c and $\mathrm{d}$ are two of the 9 factors, real space and diffraction pattern with an overlapping region. Fig. 1e is the raw diffraction pattern form the region indicated in the real-space images in c and d. Figure 2 is a sample STEM diffraction pattern taken with the direct electron camera where one frame is $1 \mathrm{msec}$. In the presentation we will discuss the challenge of analysis of these much larger (>130Gb) STEM diffraction data sets.

[1] Mott, R.B.\& Friel, J.J. (1999) J Microsc-Oxford 193, 2-14.

[2] A.J. Schwartz, et al., (2000) Electron Backscatter Diffraction in Materials Science, Kluwer Academic, New York.

[3] Kotula, P.G., Keenan, M.R. \& Michael, J.R. (2003). Microsc Microanal 9, 1-17. 
[4] Brewer, L.N., Kotula, P.G., \& Michael, J.R. (2008) Ultramicroscopy 108, 567-578.

[5] Rauch, E.F. \& Veron, M. (2005) Mat.-wiss. u. Werkstofftech. 36, 552-556.

[6] Rauch, E.F., et al. (2010) Z. Kristallogr. 225, 103-109.

[7] Ryll, H., et al., (2013) Microscopy and Microanalysis 19 1160-1161.

[8] Keenan, M.R. (2009) Surf. Int. Anal. 41, 79-87.

[9] Sandia National Laboratories is a multi-program laboratory managed and operated by Sandia Corporation, a wholly owned subsidiary of Lockheed Martin Corporation, for the US Department of Energy's National Nuclear Security Administration under contract DE-AC04-94AL85000.

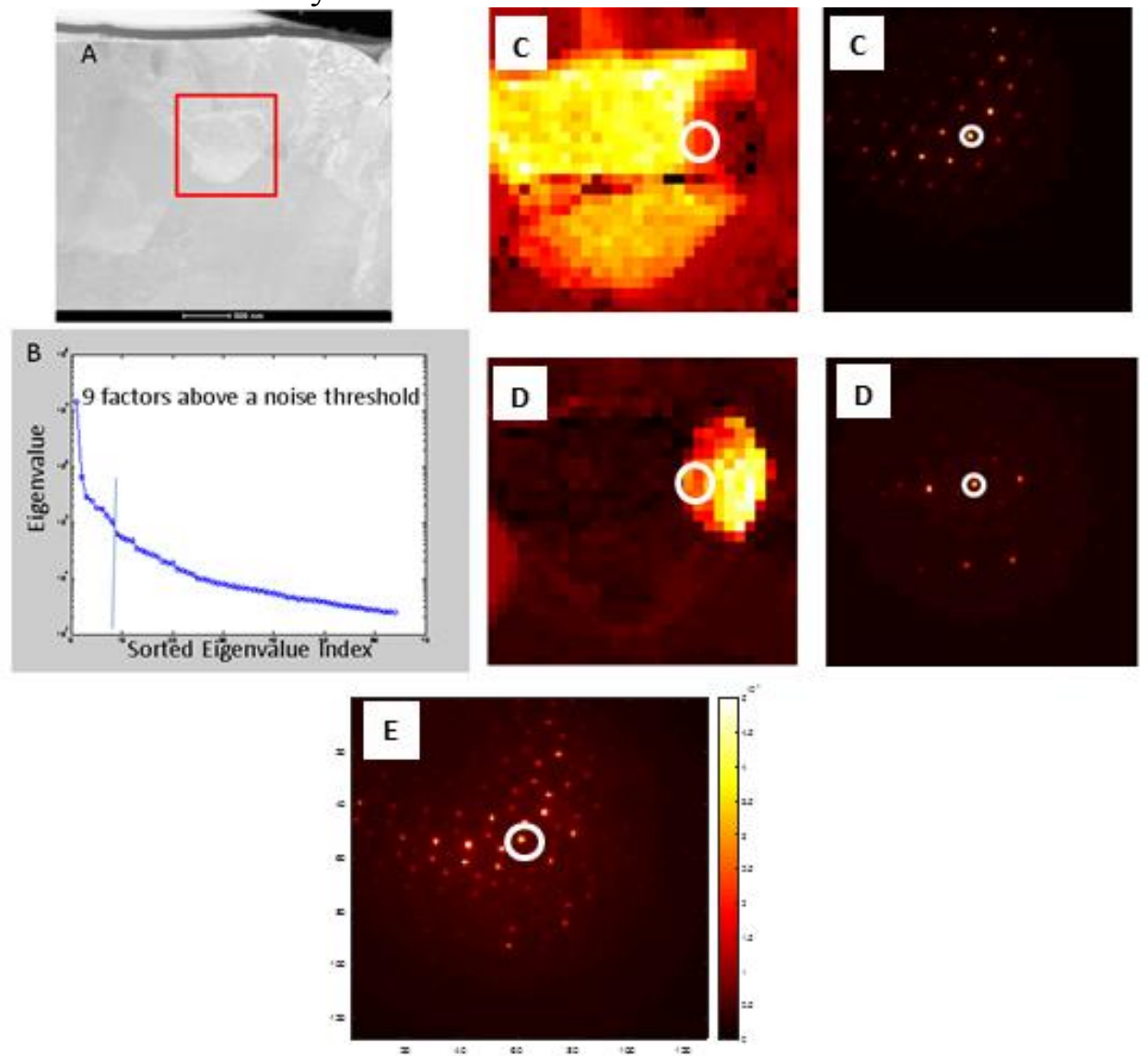

Figure 1. MSA of the diffraction data set. A. STEM overview image. B. Scree plot showing a prominent break-point after the $9^{\text {th }}$ factor. C and D. Two factors, image and corresponding diffraction pattern, with an overlapping region indicated by the circle in the real-space images. E. Raw diffraction pattern from the mixed pixel.
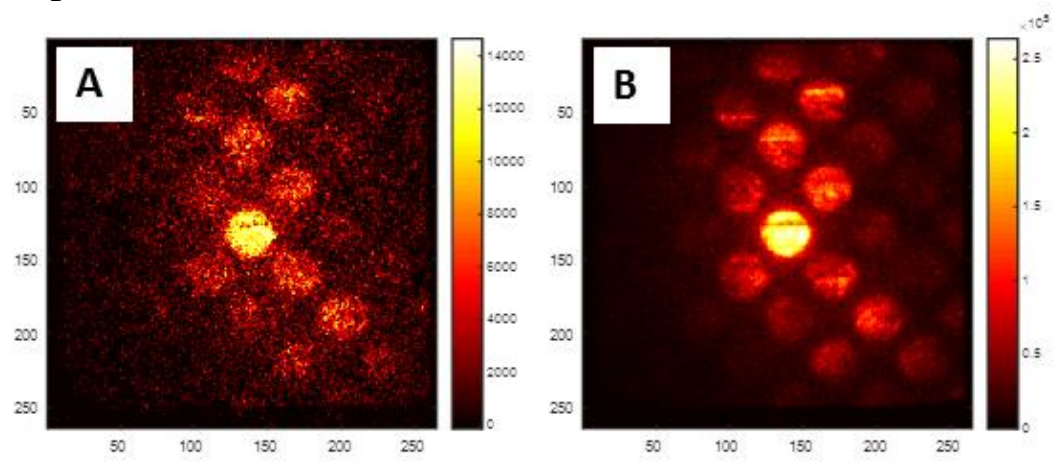

Figure 2. STEM diffraction pattern taken with the direct electron sensor. A. 1 frame, B. 20 frames. 